\title{
Detailed Analysis of High Frequency Auditory Brainstem Response in Patients with Tinnitus: A Preliminary Study
}

\author{
Joseph Pinkl \\ Matthew J Wilson \\ Danica Billingsly ${ }^{1}$ \\ Raymundo Munguia-Vazquez ${ }^{1,2}$
}

\begin{abstract}
Increased spontaneous activity and aberrant neural synchrony is thought to be the underlying cause of tinnitus. The perceived pitch of tinnitus may be dictated by frequency specific neural fibers of the subcortical pathway, or the projection of altered cortical activity by-way-of tonotopic reorganizations. Subcortical neural activity in relation to tinnitus was characterized using ABR measurements. In the present study, 11 patients (21 ears) with constant tonal tinnitus underwent a two-part experiment. Experiment 1 involved click ABR measurements and included two experimental groups: tinnitus with normal hearing from $2000-4000 \mathrm{~Hz}(\mathrm{Gl})$ and tinnitus with hearing loss within the range of $2000-4000$ $\mathrm{Hz}$ (GII). Experiment 2 utilized tone burst ABRs matched to each participant's perceived tinnitus pitch and included two experimental groups: tinnitus with normal hearing at the tinnitus pitch (Gla) and tinnitus with hearing loss at the tinnitus pitch (Glla). These groups were compared to a control group (GIII) of ten monaurally tested (10 ears) participants with normal hearing thresholds at $250-20000 \mathrm{~Hz}$ and no tinnitus. Click ABR results indicate significantly prolonged V-III IPLs for GI and GIl and a significantly extended absolute V latency for Gll only. Tone burst ABRs matched to tinnitus pitch revealed significantly prolonged absolute latencies and IPLs at three of the seven frequencies for Glla. ABR threshold seeking was completed and revealed negative eHL values for two of the four different stimuli for Gl and Gla and four of the eight stimuli for GIl and Glla. Click ABRs results are suggestive of upper brainstem abnormalities for both groups. While GI demonstrated prolonged V-III IPLs, no significant differences were found for Gla. This suggests that there is no frequency specific subcortical characteristic associated with tinnitus with normal hearing. Frequency specific properties for subcortical activity could not be characterized due to varying results of Glla.
\end{abstract}

Keywords: tinnitus, ABR, neural synchrony, spontaneous neural activity, tonotopic reorganization, inferior colliculus.

Abbreviations: Auditory brainstem response, ABR; auditory cortex, AC; centimeters cubed, $\mathrm{cm}^{3}$; decapascal, daPa; decibels, dB; gamma-aminobutyric acid, GABA; estimated hearing level, eHL; hearing level, $\mathrm{HL}$; hertz, Hz; inferior colliculus, IC; interpeak latency, IPL; normalized hearing level, $\mathrm{nHL}$; second, sec; sensation level, SL; Tinnitus Handicap Inventory, $\mathrm{THI}$

${ }^{1}$ School of Allied Health and Communication Disorders, Northern Illinois University, DeKalb, Illinois

${ }^{2}$ Indiana University School of Medicine-West Lafayette / Speech, Language \& Hearing Sciences, Purdue University, West Lafayette, Indiana

Institution: School of Allied Health and Communication Disorders, Northern Illinois University

Send correspondence to:

Joseph Pinkl

Steeple Run Crystal Lake IL, 60014, USA, E-mail: pinkljt@mail.uc.edu

Paper submitted to the ITJ-EM (Editorial Manager System) on March 20, 2017;

and accepted on April 06, 2017. 


\section{INTRODUCTION}

Tinnitus is the conscious perception of sound that is not generated by any exterior stimulation. It is considered a symptom that is commonly associated with audiologic conditions including sensorineural hearing loss $^{1-3}$. Diminished peripheral input caused by hearing loss, particularly sloping configurations can lead to reorganizations of the tonotopic framework of the $A C$ through alterations of pyramidal synaptic connections and reduced GABA output within the cortical neural infrastructure ${ }^{4-7}$. Such tonotopic reorganizations over represent neural activity near tonotopic regions of the "edge" frequency or midpoint of the hearing loss slope $e^{2,4,8,9}$. When neural activity within a selected region of the cortical tonotopic map is diminished, synaptic connections of the affected regions merge with stronger neighboring neural areas which lead to this overrepresentation of "edge" frequencies ${ }^{10}$. The over-representation of cortical activity within a specific region of the tonotopic map naturally promotes neural synchronization potentially causing a phantom perception. Furthermore, it is suspected that adaptive changes in the $\mathrm{AC}$ can alter its connections with the IC making it more susceptible to incoming afferent spontaneous signals, enhancing tinnitus perception ${ }^{11}$.

There are descending auditory pathways that serve as major connections between the cortical areas and the lemniscal and nonlemniscal systems ${ }^{12,13}$. These structures generate multiple feedback loops that extract meaning from afferent stimulation through modulation ${ }^{14,15}$. Cortical stimulation affects the tuning curves of subcortical structures causing specific regions in the subcortical system to become robustly tuned to higher levels of activity ${ }^{16-18}$. Because electrical stimulation of cortical regions activates auditory efferent functions, it is speculated that plastic alterations of inhibition and excitation within the tonotopically remapped cortex may also change the tuning curves of subcortical structures including the thalamus and IC, perhaps strengthening the attention to tinnitus and its associated frequency ${ }^{1,5}$. However, tonotopic reorganizations and altered efferent activity may not be present in all cases of tinnitus. Functional MRIs performed by Langers, de Kleine and van Dijk (2012) indicated that macroscopic tonotopic reorganization is not common for tinnitus patients with normal hearing thresholds ${ }^{19}$. Furthermore, IC neural recordings performed by Bauer et al. (2008) revealed significantly increased contralateral IC spontaneous spiking and cross fiber synchrony in chinchillas with tinnitus (as indicated by behavioral paradigms) with no evidence of a tonotopic edge effect or tuning frequency shift ${ }^{20}$. A separate viewpoint identifies tinnitus as a result of homeostatic plasticity at the subcortical level to reciprocate reduced afferent input of the periphery: the subcortical neural synchrony model.

Reduction of external stimulation as a result from hearing loss may disrupt homeostasis of a receptive neuron which can lead to alterations of inhibition factors at the brainstem level as a means of compensation to upregulate neural activity, possibly leading to a phantom stimulation ${ }^{5,21-23}$. The increase of spontaneous firing rates is inversely correlated with the neural inhibition factor ${ }^{24}$. Increases in hearing thresholds are believed to be correlated with reduced inhibition through a declination of glycine and feedforward inhibition provided by dorsal cochlear nucleus cartwheel cells, affecting neuronal homeostasis $5,25,26$. Single unit studies of fusiform cells in the dorsal cochlear nucleus indicate increased spontaneous spike rates in chinchillas that exhibited behavioral evidence of tinnitus ${ }^{27}$. These cochlear nucleus fusiform cells feed directly into the central nucleus of the $\mathrm{IC}^{20}$. The generation of constant tinnitus may be the product of a "domino effect" caused by aberrant increases of spontaneous neural output starting at the site of the cochlear nucleus which works up to the IC and eventually to the diencephalon ${ }^{20}$.

About $10 \%$ of tinnitus patients have normal behavioral thresholds ${ }^{28}$. These unique cases of tinnitus may comply with the same mechanisms of the neural synchrony model. In 2009, Kujawa and Liberman demonstrated permanent effects of peripheral neural degeneration at basal end regions by-way-of auditory brainstem response (ABR) suprathreshold amplitude measurements following recovery of temporary noiseinduced threshold shifts in the mouse model ${ }^{29}$. Further microscopy revealed an immediate decrease of synaptic ribbon counts and a delayed onset of reduced ganglion cell density (after 64 weeks) at these regions with intact outer hair cell components. This effect is localized to low and medium spontaneously active fibers $(<18$ spikes/sec) ${ }^{30-32}$. There are multiple synaptic connections associated with each inner hair cell, each constituting a specific spontaneous firing rate thus resulting in different intensity thresholds (low and medium spontaneously active fibers are reserved for detecting loud and medium level sound inputs respectively, whereas high spontaneous fibers dictate perception of soft inputs). The consequences of this peripheral neural degeneration is not a decrease of hearing sensitivity, but likely a reduction of intensity discriminating abilities, peripheral loudness growth functions and temporal coding necessary for hearing in the presence of interfering background noise a phenomenon known as noise induced cochlear synaptopathy or "hidden hearing loss"23,31-35. Because hidden hearing loss involves a systematic decrease of peripheral auditory nerve output, it is speculated that homeostatic upregulation takes effect in the rare cases of tinnitus with normal hearing thresholds ${ }^{23,36}$.

In the case of tinnitus accompanied by hearing loss, it is believed that tinnitus pitch is either localized in the "edge" frequencies or within the lowest regions of the hearing loss ${ }^{19}$. If tinnitus pitch exists within the lowest portions of the corresponding hearing loss, then theoretically, specific subcortical neural fibers act as the direct factor of tinnitus percept ${ }^{19}$. However, if tinnitus percept exists at the "edge" frequency, alterations of the 
tonotopic map would be implicated as the primary source of tinnitus percept, possibly supplemented by affected IC tuning curves ${ }^{19}$. Abnormal synchronous neural activity can be detected by specialized clinical tests. Though these tests are not a definitive diagnostic tool for tinnitus, potentially they can be used to characterize subcortical activity in relation to tinnitus ${ }^{37,38}$.

We propose a tinnitus ABR study utilizing click stimuli and tinnitus pitch matched tone burst stimuli to objectively measure the electrophysiological activity generated within subcortical levels of the auditory pathway. Analyzing waveform measurements of the ABR may help localize hyperactivity and/or hypoactivity to specific regions of the subcortical pathway including the vestibulocochlear nerve, dorsal cochlear nucleus, superior olivary complex, lateral lemniscus and $\mathrm{IC}^{39}$.

Understanding the role of the subcortical areas in tinnitus can influence future treatment approaches. If activity of the lower subcortical pathway (below the IC) is primarily responsible for tinnitus perception, management strategies should focus on partially masking the tinnitus (i.e. tinnitus masker devices). If tinnitus percept is primarily influenced by abnormal cortical activity, treatment should aim to alter the cortical tonotopic mapping structure in tinnitus patients through the use of sound enrichment and training ${ }^{7,40}$. Furthermore, identifying potential correlations between $A B R$ readings and tinnitus pitch can help formalize tinnitus diagnostic procedures. Such procedures will be useful for forensic evaluations for veterans and industrial workers. Considering the models of pathologic enhanced neural synchronization and the potential cortical influence on subcortical tuning functions, it is hypothesized that unique $A B R$ readings if any, will become more pronounced in tinnitus subjects if the ABR parameters are adjusted from click stimuli to tone burst stimuli matched to the tinnitus pitch. Specifically, we expect to see at least a distinct wave $V$ reading as this wave is generated by higher processing regions with high natural spontaneous activity and strong connections to the cortex and other subcortical structures.

\section{MATERIALS AND METHODS}

\section{Participants}

Prior to any testing, informed consent was obtained and documented from all participants. Approval for all data collection procedures was obtained from the University's Institutional Review Board before initiating the study. 11 participants for a total of 21 tested ears (one participant reported unilateral tinnitus) with constant tonal tinnitus were recruited through local private audiology practices and the University Audiology Clinic (range: 18-75 years; mean \pm 1 standard deviation: 46.48 years \pm 19.15 ). These patients participated in a two part experiment. Experiment 1 involved click ABR measurements for two experimental groups: individuals with tinnitus and normal hearing within the frequency range of $2000-4000 \mathrm{~Hz}(\mathrm{Gl})$, participants with tinnitus and hearing loss within the range of $2000-4000 \mathrm{~Hz}$ (GII). Audiometric criteria for hearing loss was only applied in the range of $2000-4000 \mathrm{~Hz}$ for these groups because this is the frequency range that best corresponds to the standard ABR click. During experiment 2 , ABR stimuli were changed from clicks to tinnitus pitch matched tone burst stimuli. Participants were re-divided into two new experimental groups: participants with tinnitus and normal hearing at the perceived frequency of the tinnitus (Gla) and participants with tinnitus and hearing loss at the perceived frequency of tinnitus (Glla). Gl consisted of a total of 10 ears, Gll consisted of 11 ears, Gla consisted of 9 ears and Glla consisted on 12 ears. A control group (GIII) (22-29 years; 24.4 years \pm 2.0 ) that included 10 subjects free from both tinnitus, hearing loss, middle ear pathology, ototoxic medications and ear surgeries was included for comparison purposes. Due to the presence of asymmetric and tinnitus and hearing thresholds among the tested sample, each ear was assessed individually. Refer to Table 1 for demographic data of each group.

\section{Case history and immittance screening}

Participants were asked to complete a case history form. The case history intake form covered information regarding medical background, surgeries, noise exposure and ototoxic medications as well as auditory related symptoms: difficulty of hearing and communication, otalgia, otorrhea, recent otitis externa/media, dizziness and aural fullness. This was used to determine eligibility of the study. Participants with medical backgrounds involving ear surgeries, ototoxic medication, and otitis externa/media and known middle ear pathologies were omitted. Middle ear air pressure and tympanic membrane compliance were measured through standard $226 \mathrm{~Hz}$ tympanometry using a GSI Tympstar Middle Ear Analyzer (Grason-Stadler). Participants who fell outside of the normative range of -100 to 50 daPa middle ear pressure and 0.3 to $1.9 \mathrm{~cm}^{3}$ compliance were deemed ineligible for the study.

\section{Acquisition of audiometric thresholds}

All eligible participants underwent behavioral audiometric threshold measurements using a GSI 61 Clinical Audiometer. Standard air and bone conducted signals were delivered via EARTone 3A insert earphones, and a MelMedtronics B71 adult bone oscillator, respectively. Extended high frequency air conduction pure tones were presented through Sennheiser HDA200 high frequency headphones. All test signals were generated through the GSI-61 audiometer. Pure tone thresholds seeking was completed using the HughsonWestlake ${ }^{41}$ method. Responses to pure tone signals were

Table 1. Number of ears (n) and average age \pm 1 standard deviation for each group. Note: GIII consisted of a total of 10 ears however normative calculations excluded uninterpretable/ unreliable ABR readings. Uninterpretable/unreliable ABRs varied from 0-2 across all ABR normative calculations.

\begin{tabular}{cccccc}
\hline & Gl & Gll & Gla & Glla & GIII \\
\hline $\mathrm{n}$ & 10 & 11 & 9 & 12 & 10 \\
age & $39.6 \pm 14.7$ & $52.7 \pm 21.2$ & $37.6 \pm 14.7$ & $52.6 \pm 20.2$ & $24.4 \pm 2.0$ \\
\hline
\end{tabular}


measured at $250 \mathrm{~Hz}, 500$ to $8000 \mathrm{~Hz}$ (at half octave steps) and the following high frequencies: 9000, 10000, 11200, 12500, 14000, 16000, 18000 and 20,000 Hz bilaterally. Thresholds of $30 \mathrm{~dB} \mathrm{HL}$ or more at any of the tested frequencies were considered to be a hearing loss at that corresponding frequency. Those with air/bone gaps of $15 \mathrm{~dB}$ or greater in two or more consecutive frequencies were removed from the study.

\section{Tinnitus handicap inventory and tinnitus pitch matching protocol}

Experimental participants were asked to complete the THI- a self-report measurement of tinnitus handicap ${ }^{42}$. These responses were calculated and recorded. Pitch matching was completed using an $A / B$ forced choice paradigm. Two random tones were presented separately $10 \mathrm{sec}$ apart for $5 \mathrm{sec}$ at time. The continuous pure tones were presented at $20 \mathrm{~dB}$ above the behavioral threshold. Each participant was tasked with identifying the tone that sounded closer to the pitch of the tinnitus. The presented tones ranged from 1000 to $20000 \mathrm{~Hz}$. Procedures were repeated until one frequency was identified. Participants who perceive multiple simultaneous tinnitus pitches were asked to consider the most prominent pitch during the pitch matching procedure. Refer to Table 2 for pitch matching results.

\section{Acquisition of auditory brainstem responses}

All ABRs were acquired under a one-channel, vertical electrode configuration, using sticker electrodes applied to the surface of the scalp. The non-inverting electrodes were placed according to the $10-20$ system $^{43}$ at electrode Fpz (high forehead). The reference and ground electrodes were placed on the left and right earlobes, respectively. Electrode impedances were measured below $5 \mathrm{k} \Omega$, and within $1 \mathrm{k} \Omega$ of each other.

During Experiment 1, click evoked ABRs were administered. $85 \mathrm{~dB} \mathrm{nHL}$ rarefaction stimuli were presented via E-A-RTONE Gold 3A insert earphones (Etymotic Research, Elk Grove Village, IL) at a rate of 21.1 stimuli/sec. An artifact rejection algorithm was applied to the on-line averaging waveform. If the peak voltage within

Table 2. Tinnitus pitch matching results for Gla and Glla and the number of ears in each frequency $(n)$. Frequencies that yielded significant ABR findings are identified $\left({ }^{*}\right)$.

Gla

\begin{tabular}{ccc}
\hline Pitch Match & n \\
\hline & $4000 \mathrm{~Hz}$ & 3 \\
$12500 \mathrm{~Hz}$ & 4 \\
& $16000 \mathrm{~Hz}$ & 2 \\
\hline Glla & \\
\hline & Pitch Match & $\mathbf{n}$ \\
\hline $1500 \mathrm{~Hz}$ & 1 \\
$4000 \mathrm{~Hz}$ & 1 \\
$6000 \mathrm{~Hz}$ & $2^{*}$ \\
$8000 \mathrm{~Hz}$ & 2 \\
$9000 \mathrm{~Hz}$ & 2 \\
$12500 \mathrm{~Hz}$ & $2^{*}$ \\
$14000 \mathrm{~Hz}$ & $2^{*}$ \\
\hline
\end{tabular}

a sweep exceeded $\pm 23.9 \mathrm{uV}$, that sweep was excluded from the averaged waveform. The ongoing average of the ABR waveform was monitored during acquisition. IHS (Intelligent Hearing System) hardware with SmartEP software was used for data acquisition. A total of 1024 sweeps were collected at a sample rate of $512 \mathrm{~Hz}$. A bandpass filter with a bandwidth of $100-3000 \mathrm{~Hz}$ and rejection rate of $-6 \mathrm{~dB} /$ octave was used. The time window for the ABR was 10.66 millisec.

For Experiment 2, tone burst evoked ABRs were performed. $85 \mathrm{~dB} \mathrm{nHL}$ rarefaction stimuli were presented via ER-2 high frequency insert headphones (Etymotic Research, Elk Grove Village, IL), augmented by an IHS Sound Output Booster box. Tone burst frequency was chosen based on pitch matched frequency and included the following center frequencies: $1500,4000,6000,8000$, $9000,12500,14000$ and $16000 \mathrm{~Hz}$ (Table 2). Total sweeps, sampling rate, time window, and filter settings were the same as Experiment 1.

Click and tone burst threshold seeking were performed on all participants, wave $\mathrm{V}$ amplitude and absolute I, III and V latencies were observed. When a V wave was identified and repeatable, stimulus intensity was decreased by $10 \mathrm{~dB}$. Procedures were repeated until the wave $V$ was no longer identifiable, and then the intensity was increased by $5 \mathrm{~dB}$. The threshold was defined as the lowest intensity level needed to record an identifiable wave $\mathrm{V}$.

During testing, all participants laid in a supine position on a test bed in an isolated testing room. They were instructed to remain still with eyes closed throughout the entire test with arms and legs uncrossed to reduce risk of myogenic interference. The participants were given breaks as necessary.

\section{Acquisition of click and tone burst normative data}

Ten total control subjects (10 ears) were tested to establish normative data. ABR stimuli were presented to the participant's right ear. The most reliable/repeatable waveforms were used in the analysis. Unrepeatable and/or uninterpretable ABR data was suspended from the normative pool. Normative click and tone burst ABR data within the control group (with consistent equipment, parameters and test environment) were calculated and compared with the experimental data.

For both click and tone burst threshold seeking, correction factors $/ \mathrm{nHL}$ values were designed by averaging the difference between the ABR threshold average and the corresponding behavioral threshold average (2000$4000 \mathrm{~Hz}$ pure tone averages were used for clicks). They were rounded up to the nearest $5 \mathrm{~dB}$ mark. Calculated correction factors are displayed in Table 3.

\section{Analysis of data}

ABR waves, I, III, and $V$ absolute latencies and V amplitudes as well as III-I, V-III, and V-I IPLs were all analyzed. Statistical analysis was performed using unpaired t-tests at each specified ABR metric using the 
IBM (International Business Machines Corporation) SPSS-V21 package. Two-tailed probabilities of less than $0.05(p<0.05)$ were considered statistically significant.

\section{RESULTS}

\section{Preliminary Testing}

THI scoring from the experimental group ranged from 12 (grade 1: mild) to 40 (grade 3: moderate). The sample had an average score of 22.8 (grade 2: mild) and a standard deviation of 8.5. The location of the matched tinnitus frequency in relation to the hearing loss configuration was observed. Of the 21 tested ears, 3 participants (total of 5 ears) experienced tinnitus pitch at the "edge" frequency and 3 participants (total of 6 ears) experienced tinnitus at the lowest portion of the hearing loss. The remaining 5 participants (total of 10 ears) fell into neither category. THI and tinnitus pitch location in relation to the audiogram are not displayed.

\section{Experiment 1}

Click ABR measurements were compared between three subject groups. GI and Gll were established based the pure tone average at 2000, 3000 and $4000 \mathrm{~Hz}$. Those with a threshold below $30 \mathrm{~dB} \mathrm{HL}$ were considered $\mathrm{Gl}$ participants. Those with a threshold at $30 \mathrm{~dB} H \mathrm{HL}$ or greater

Table 3. Established $\mathrm{nHL}$ values calculated from GIII.

\begin{tabular}{cc}
\hline Frequency & Correction Factor \\
\hline Click & $-15 \mathrm{~dB}$ \\
$1500 \mathrm{~Hz}$ & $-35 \mathrm{~dB}$ \\
$4000 \mathrm{~Hz}$ & $-25 \mathrm{~dB}$ \\
$6000 \mathrm{~Hz}$ & $-30 \mathrm{~dB}$ \\
$8000 \mathrm{~Hz}$ & $-35 \mathrm{~dB}$ \\
$9000 \mathrm{~Hz}$ & $-40 \mathrm{~dB}$ \\
$12500 \mathrm{~Hz}$ & $-50 \mathrm{~dB}$ \\
$14000 \mathrm{~Hz}$ & $-55 \mathrm{~dB}$ \\
$16000 \mathrm{~Hz}$ & $-55 \mathrm{~dB}$ \\
\hline
\end{tabular}

were grouped in GII. Using this approach, GI contained a total of 10 ears, Gll contained 11 ears and GIII contained 10 ears. When comparing Gl with GIII, there were no statistically significant findings pertaining to absolute wave I, III and V latencies, wave V amplitude, IPL III-I, and IPL V-I. The IPL V-III was significantly prolonged in GI ( $p$ $<0.05)$ Table 4.

ABR measurements of GIl in comparison to GIII indicated no statistically significant findings pertaining to absolute latencies of wave I and III, wave $\mathrm{V}$ amplitude, IPL III-I and IPL V-I. Absolute wave V latencies of Gll were significantly delayed and IPL V-III of GII were significantly prolonged $(p<0.05)$ Table 5.

Comparisons of $\mathrm{Gl}$ and GII revealed no statistically significant findings involving absolute wave I, III and V latencies, wave V amplitudes, IPL III-I and IPL V-III. The IPL V-I was significantly delayed in GI $(p<0.05)$ Table 6.

\section{Experiment 2}

Participants were re-divided based on hearing thresholds at the determined pitch of tinnitus. Gla included a total of nine ears and Glla included 12 total ears (Table 2). Three (total of six ears) of the seven frequencies tested for Glla yielded significant differences $(p<0.05)$. At 6000 $\mathrm{Hz}$, Glla presented a significantly delayed the absolute V latency compared to GIII. At $12500 \mathrm{~Hz}$, Glla presented significantly delayed absolute III and $\mathrm{V}$ waves compared to the GIII normative. Finally at $14000 \mathrm{~Hz}$, Glla yielded significantly delayed absolute wave I and $\mathrm{V}$ latencies as well as significantly prolonged IPL V-III and IPL V-I. Complete findings for these three tone-burst ABRs are displayed in Table 7.

\section{ABR thresholds}

Using calculated correction factors (Table 3), intensity levels at $\mathrm{dB} S \mathrm{SL}$ were calculated to estimate the lowest $S L$ of the $A B R$ stimulus required for a response.

Table 4. Comparison between absolute latencies, IPLs and amplitude mean \pm 1 standard deviation of GI and GIII. Significant findings are identified ( $\left.{ }^{*}\right)$.

\begin{tabular}{ccccc}
\hline ABR Measurement & Group I & Group III & t-Test & Sig. (2 tailed) \\
\hline I Latency & $1.723 \pm 0.102$ & $1.556 \pm 0.102$ & -1.931 & 0.069 \\
III Latency & $3.699 \pm 0.405$ & $3.761 \pm 0.148$ & 0.455 & 0.655 \\
V Latency & $5.604 \pm 0.328$ & $5.509 \pm 0.144$ & -0.840 & 0.412 \\
V Amplitude & $.538 \pm 0.369$ & $0.5110 \pm 0.155$ & -0.214 & 0.833 \\
IPL III-I & $2.066 \pm 0.506$ & $2.021 \pm 0.166$ & 0.831 & 0.417 \\
IPL V-III & $1.906 \pm 0.441$ & $1.751 \pm 0.199$ & -1.012 & $0.0331^{*}$ \\
IPL V-I & $3.836 \pm 0.174$ & $3.954 \pm 0.183$ & 1.477 & 0.157 \\
\hline
\end{tabular}

Table 5. Comparison between absolute latencies, IPLs and amplitude mean \pm 1 standard deviation of GII and GIII. Significant findings are identified $\left({ }^{\star}\right)$.

\begin{tabular}{ccccc}
\hline ABR Measurement & Group II & Group III & t-Test & Sig. (2 tailed) \\
\hline I Latency & $1.581 \pm 0.322$ & $1.556 \pm 0.102$ & -0.244 & 0.812 \\
III Latency & $3.625 \pm 0.364$ & $3.761 \pm 0.148$ & 1.145 & 0.272 \\
V Latency & $5.811 \pm 0.383$ & $5.509 \pm 0.144$ & -2.431 & $0.03^{\star}$ \\
V Amplitude & $0.411 \pm 0.140$ & $.511 \pm 0.155$ & 1.553 & 0.137 \\
IPL III-I & $2.046 \pm 0.258$ & $2.021 \pm 0.166$ & 1.664 & 0.113 \\
IPL V-III & $2.186 \pm 0.562$ & $1.751 \pm 0.199$ & -2.409 & $0.032^{\star}$ \\
IPL V-I & $4.230 \pm 0.485$ & $3.954 \pm 0.183$ & -1.755 & 0.103 \\
\hline
\end{tabular}


Table 6. Comparison between absolute latencies, IPLs and amplitude mean \pm 1 standard deviation of GI and GII. Significant findings are identified $\left({ }^{*}\right)$.

\begin{tabular}{ccccc}
\hline ABR Measurement & Group I & Group II & t-Test & Sig. (2 tailed) \\
\hline I Latency & $1.723 \pm 0.102$ & $1.581 \pm 0.322$ & 1.116 & 0.278 \\
III Latency & $3.699 \pm 0.405$ & $3.625 \pm 0.364$ & 0.444 & 0.662 \\
V Latency & $5.604 \pm 0.328$ & $5.810 \pm 0.383$ & -1.323 & 0.201 \\
V Amplitude & $.538 \pm 0.369$ & $0.411 \pm 0.1404$ & 1.025 & 0.327 \\
IPL III-I & $2.066 \pm 0.506$ & $2.046 \pm 0.258$ & 0.114 & 0.911 \\
IPL V-III & $1.906 \pm 0.441$ & $2.186 \pm 0.562$ & -1.262 & 0.222 \\
IPL V-I & $3.836 \pm 0.174$ & $4.230 \pm 0.485$ & -2.510 & $0.026^{*}$ \\
\hline
\end{tabular}

Table 7. Comparison between absolute latencies, IPLs and amplitude mean \pm 1 standard deviation of Glla and GIII at tone-burst ABR frequencies of 6000,12500 and $14000 \mathrm{~Hz}$. Significant findings are identified ( ${ }^{*}$ ).

\begin{tabular}{|c|c|c|c|c|c|}
\hline $\mathrm{Fz}$ & ABR Measurement & Group Ila & Group III & $\mathrm{t}$-Test & Sig. (2 tailed) \\
\hline \multirow[b]{6}{*}{$6000 \mathrm{~Hz}$} & I Latency & $2.175 \pm 0.213$ & $1.900 \pm 0.438$ & -0.829 & 0.434 \\
\hline & III Latency & $3.750 \pm 0.354$ & $4.164 \pm 0.683$ & -0.800 & 0.450 \\
\hline & V Latency & $5.585 \pm 0.545$ & $6.557 \pm 0.340$ & -3.225 & $0.015^{*}$ \\
\hline & V Amplitude & $0.190 \pm 0.2404$ & $0.333 \pm 0.125$ & -1.209 & 0.266 \\
\hline & IPL III-I & $1.575 \pm 0.573$ & $2.266 \pm 0.847$ & -1.059 & 0.325 \\
\hline & IPL V-III & $1.835 \pm 0.191$ & $2.393 \pm 0.748$ & -1.000 & 0.351 \\
\hline & IPL V-I & $3.415 \pm 0.757$ & $4.659 \pm 0.738$ & -2.094 & 0.075 \\
\hline \multirow{10}{*}{$12500 \mathrm{~Hz}$} & I Latency & $2.475 \pm 0.460$ & $1.386 \pm 0.149$ & 3.302 & 0.176 \\
\hline & III Latency & $4.580 \pm 0.424$ & $3.124 \pm 0.487$ & 3.769 & $0.007^{\star}$ \\
\hline & V Latency & $6.780 \pm 0.141$ & $4.791 \pm 0.667$ & 4.001 & $0.005^{\star}$ \\
\hline & V Amplitude & $0.240 \pm 0.0707$ & $.210 \pm 0.131$ & 0.301 & 0.772 \\
\hline & IPL III-I & $2.105 \pm 00.035$ & $1.700 \pm 0.522$ & 1.044 & 0.331 \\
\hline & IPL V-III & $2.200 \pm 0.566$ & $1.664 \pm 0.539$ & 1.230 & 0.258 \\
\hline & IPL V-I & $4.300 \pm 0.594$ & $3.406 \pm 0.777$ & 1.480 & 0.182 \\
\hline & I Latency & $1.485 \pm 0.304$ & $1.076 \pm 0.173$ & 2.590 & $0.036^{\star}$ \\
\hline & III Latency & $3.165 \pm 0.120$ & $2.660 \pm 0.393$ & 1.172 & 0.130 \\
\hline & V Latency & $6.607 \pm 0.332$ & $4.710 \pm 0.651$ & 2.745 & $0.024^{*}$ \\
\hline \multirow{4}{*}{$14000 \mathrm{~Hz}$} & V Amplitude & $0.550 \pm 0.184$ & $.269 \pm 0.210$ & 1.702 & 0.133 \\
\hline & IPL III-I & $1.680 \pm 0.424$ & $1.584 \pm 0.377$ & 0.311 & 0.765 \\
\hline & IPL V-III & $2.900 \pm 0.453$ & $1.926 \pm 0.426$ & 2.825 & $0.026^{\star}$ \\
\hline & IPL V-I & $4.575 \pm 0.035$ & $3.511 \pm 0.651$ & 4.301 & $0.005^{\star}$ \\
\hline
\end{tabular}

Negative sensation levels were calculated at 2 of the 4 ABR stimuli for Gl and Gla. This included 12500 and $14000 \mathrm{~Hz}$. Negative sensation levels were recorded at 4 of the 8 ABR stimuli for Gll and Glla. This included 1500, 6000, 8000 and $9000 \mathrm{~Hz}$; Table 8.

\section{DISCUSSION}

Click ABRs results are suggestive of upper brainstem abnormalities for both Gl and GIl. As stated earlier, it is theorized that spontaneous activity and tuning curves of the IC and other upper brainstem structures may be correlated with changes of the AC tonotopic map. If tuning curves of higher level subcortical structures are concentrated at frequency regions associated with tinnitus perception, synchronized neural activity would be expected to be much higher in those specific regions. Increased wave $\mathrm{V}$ amplitudes and a possible decrease of absolute $V$ latencies were expected as these findings would be consistent with enhanced neural synchrony of the upper brainstem ${ }^{44}$. This however was not observed. The prolonged V-III IPL may be explained by the "baseline" theory of spontaneous neural activity. The "baseline" theory of spontaneous auditory activity identifies such activity as a baseline configuration of the
Table 8. Calculated ABR thresholds (dB SL) for each experimental group. The $\mathrm{nHL}$ data from GIII (Table 3) were used to calculate findings.

Gl and Gla

\begin{tabular}{cc}
\hline Frequency & ABR Threshold \\
\hline Click & $9 \mathrm{~dB} \mathrm{SL}$ \\
$4000 \mathrm{~Hz}$ & $15 \mathrm{~dB} \mathrm{SL}$ \\
$12500 \mathrm{~Hz}$ & $-18 \mathrm{~dB} \mathrm{SL}$ \\
$16000 \mathrm{~Hz}$ & $-18 \mathrm{~dB} \mathrm{SL}$ \\
\hline Gll and Glla & \\
\hline Frequency & ABR Threshold \\
\hline Click & $11 \mathrm{~dB} \mathrm{SL}$ \\
$1500 \mathrm{~Hz}$ & $-10 \mathrm{~dB} \mathrm{SL}$ \\
$4000 \mathrm{~Hz}$ & $0 \mathrm{~dB} \mathrm{SL}$ \\
$6000 \mathrm{~Hz}$ & $-15 \mathrm{~dB} \mathrm{SL}$ \\
$8000 \mathrm{~Hz}$ & $-21 \mathrm{~dB} \mathrm{SL}$ \\
$9000 \mathrm{~Hz}$ & $-13 \mathrm{~dB} \mathrm{SL}$ \\
$12500 \mathrm{~Hz}$ & $0 \mathrm{~dB} \mathrm{SL}$ \\
$14000 \mathrm{~Hz}$ & $5 \mathrm{~dB} \mathrm{SL}$ \\
\hline
\end{tabular}

nervous system ${ }^{45}$. Sound perception is generated by modulations of the pre-established spontaneous firing rates $^{45}$. Essentially, spontaneous activity serves as a carrier of information; without it, the perception of sound 
could not exist. If modulations of the baseline dictate sound perception, it is possible that tinnitus causes an increased spontaneous baseline that can interfere with external stimulation thus prolonging ABR latencies. Our findings should be further investigated through animal model spontaneous neural measurements at the IC level with supplemental multifocal microscopy.

While Gl demonstrated significantly prolonged IPL V-III, no significant differences for the ABR analyses were found during tone burst ABR testing for Gla. This suggests that there is no frequency specific characteristic associated with subcortical spontaneous activity within the tinnitus with no hearing loss population. It is possible that tinnitus pitch in patients with normal hearing thresholds is controlled by cortical mechanics separate from subcortical activity; or the ABR metrics analyzed in the present study are simply not sensitive to tinnitus related subcortical functional changes. Electrophysiology tests addressing higher level structures (i.e. mid latency response, double click P50) could be the focus of future related research along with neuroimaging testing.

Glla tone-burst $A B R$ findings were unexpected as it did not strongly relate to GIl click ABR findings. Three of the seven tone burst ABRs displayed absolute latency abnormalities however the types of abnormalities varied between each ABR tone burst frequency. Abnormal IPL $V$-III values were a common observation during click $A B R$ measurements, however this finding was only observed at one $A B R$ tone burst frequency. Considering the current data, it is impossible to make any conclusions regarding frequency specific properties of subcortical spontaneous neural activity; however, it is clear that abnormalities exist within both tests. Larger scale studies are warranted.

Perhaps the most unexpected finding of this study was significantly reduced $\mathrm{eHL}$ values within the experimental groups. This is particularly unexpected considering the traditional verification measures of tinnitus in animal studies. Tinnitus animal studies tend to rely on inhibited reflex based behavioral paradigms as a correlate to tinnitus ${ }^{46}$. The acoustic startle reflex, an unconscious defensive response to sudden loud sounds ${ }^{47}$, can be reduced by pre-pulse inhibition- weaker stimulation reducing the response of a subsequent startling stimulus ${ }^{48}$. Additionally, brief, silent gaps within a consistent noise can further inhibit the acoustic startle reflex- a phenomenon called gap pre-pulse inhibition ${ }^{49}$. This gap pre-pulse inhibition is known to be reduced in patients with tinnitus. This method has been supported and quantified in human subjects with tinnitus by measuring eye blink amplitudes via myogenic potential measurements of orbicularis oculi muscles during maximum acoustic startling and pre-pulse gap inhibited startling conditions ${ }^{50}$. Detecting reduced gap pre-pulse inhibition has become a conventional method for confirming tinnitus in animal research subjects. It was initially believed that this factor was caused by a reduced perception of gaps within a pre-pulse signal due to the constant presence of tinnitus. If tinnitus effectively "fills in" the gaps and reduces gap pre-pulse inhibition, we would expect it to have a similar effect on the time locked $A B R$ recordings and ultimately increase threshold measurements. However previous humanstudies involving subjective gap detection testing revealed no difference of subjective gap detection between age-matched tinnitus participants and non-tinnitus controls ${ }^{51,52}$. This was done by performing subjective gap detection tests with stimuli at varying intensity levels that were matched and unmatched to each participant's tinnitus pitch ${ }^{51,52}$. Initially it was believed that these findings were a contradiction to other studies that supported reduced gap pre-pulse inhibition in tinnitus patients ${ }^{53}$, although subjective gap detecting and acoustic startle reflex suppression may follow two different mechanisms. It is proposed that prepulse inhibition to the acoustic startle reflex is influenced by subcortical regions and reflexive motor circuits whereas conscious gap detection involves cortex activity ${ }^{54,55}$. From a physiological standpoint, tinnitus may not effectively "fill in" the gaps, rather, it provides an inhibition or habituation factor for certain involuntary functions such as the startle reflex. We propose a double click P50 study to investigate this potential habituation factor. Furthermore, cortical stimulation shifts IC neurons of unmatched frequencies toward the activated regions of the cortex ${ }^{16}$ which may affect ABR recordings. We speculate that some courses of tinnitus will enhance frequency specific corticofugal feedback activity, serving as a major component to its procurement $^{5}$. If altered feedback loops emphasize the tinnitus-related frequencies while inhibiting surrounding frequencies ${ }^{5}, A B R$ wave $V$ thresholds may be uniquely reduced if the stimulus frequency is matched to the subject's tinnitus.

\section{LIMITATIONS AND FUTURE AIMS}

This research was limited by the number of both experimental and control participants. First and foremost, this research should be continued on a much larger scale with larger sample sizes. While specific measures were taken to eliminate risk of ABR artifact/interpretation error, it cannot be definitively ruled out. High frequency ABRs exceeding $6000 \mathrm{~Hz}$ generated difficult to interpret waveforms. Larger samples will help reduce type I and II error. It should also be noted that the control group and experimental groups were not age matched. This was extremely difficult to do given the high prevalence of tinnitus in the elderly population and the low abundance of elderly individuals with normal high frequency hearing. Lastly, a large scale correlation study should be implemented. Tinnitus pitch in relation to the hearing configuration (edge vs. dip) should be measured and compared to various patient demographics: age, gender, tinnitus onset and duration, and THI scores as both individual correlates and regression analyses.

Notable computational and electrophysiology tinnitus studies have identified reduced wave I amplitudes with unaffected $\mathrm{V}$ amplitudes in patients with tinnitus 
and normal hearing thresholds ${ }^{23,56,57}$. This is consistent with the reduced synaptic connections of the cochlea and peripheral nerve seen in hidden hearing loss. In contrast, Guest et al. (2017) found no evidence of reduced wave I amplitudes in young adults with tinnitus and normal audiograms and no correlation between electrophysiologic measurements and degree of lifetime noise exposure ${ }^{58}$. Taking this into account, we propose future studies focusing on wave V:I amplitude ratio's to address peripheral auditory nerve activity in tinnitus patients with and without normal hearing thresholds. Correlational analyses involving noise exposure histories, tinnitus severity and V:I measurements should be included. Additionally, compound action potential (CAP) measurements utilizing ipsilateral "masked" electrocochleography (ECochG) procedures would provide a thorough examination of the peripheral nerve. Each inner hair cell in the cochlea inherits multiple synaptic connections with each fiber constituting a specific spontaneous firing rate ${ }^{30}$. Intensity coding is determined by the spontaneous firing rates of a neural fiber and theoretically, different fibers can be masked during ECochG recordings to obtain fiber specific threshold recordings. Earl and Chertoff (2012) utilized a method of auditory nerve assessment through ipsilateral high pass masking paradigms while recording CAPs to formulate neural firing density functions ${ }^{59}$. A similar recording approach can be adapted but instead of using masking functions at the frequency domain, it should be implemented at the intensity domain. CAP threshold recordings with the use of ipsilateral narrowband noise masking paradigms set at different intensity levels will isolate and evaluate the integrity of different intensity sensitive neurons within selected cochlear regions ${ }^{60}$. Potentially this will enable precise localizations of affected neural fibers in tinnitus patients.

The wide range of tinnitus pitch data and the associated tone-burst ABR normative calculations contraindicated use of analytical grouping techniques (i.e. analysis of variances). To address this set back, experimental groups separated based on the pitch of tinnitus should be considered; however, given the subjective nature of pitch matching tinnitus assessment, this will be difficult. The exact pitch of tinnitus is impossible to precisely calculate given the limitations of test equipment and patient compliance. Nonetheless, we recognize potential in this approach as this will reduce risk of type 1 error.

As mentioned earlier, there are opportunities to utilize other tests including cortical electrophysiology testing and neural imaging and confocal imaging. Using two separate collection methods will help differentiate and/or relate cortical and subcortical characteristics. Additionally, correlations between tone burst ABR thresholds, P50 suppression measurements, gap detection testing and pre-pulse inhibition using stimuli matched to tinnitus pitch can be used to further characterize subcortex and $A C$ activity. We also advise measuring ABR thresholds on two populations: tinnitus with hyperacusis and tinnitus without hyperacusis to study the effect of hyperacusis on threshold seeking and ABR I:V amplitude ratios.

This research serves as a preliminary project to investigate. Given the collective evidence concerning cortical and subcortical neural activity in relation to tinnitus, future related studies are supported. Continuity of this methods approach with the aforementioned adjustments and additions should be considered.

\section{RESEARCH HIGHLIGHTS}

- Click ABR findings are consistent with upper brainstem abnormalities for GI and Gll.

- Frequency specific properties for subcortical neural activity cannot be characterized.

- Excessively low eHL values were identified throughout all experimental groups.

\section{ACKNOWLEDGEMENT}

We thank the participants for their cooperation and time.

\section{REFERENCES}

1. Jastreboff PJ. Phantom auditory perception (tinnitus): mechanisms of generation and perception. Neuroscience Research.1990;8(4):221-54.

2. Konig O, Schaette R, Kempter R, Gross M. Course of hearing loss and the occurrence of tinnitus. Hear Res. 2006;221:59-64.

3. Sereda M, Hall DA, Bosnyak DJ, Edmondson-Jones M, Roberts LE, Adjamian P, et al. Re-examining the relationship between audiometric profile and tinnitus pitch. Int J Audiol. 2011;50:303-12.

4. Eggermont JJ. Central tinnitus. Auris Nasus Larynx. 2003;30:S7-S12.

5. Eggermont JJ. The neuroscience of tinnitus. United Kingdom: Oxford Press; 2012.

6. Muhlnickel W, Elbert T, Taub E, Flor H. Reorganization of auditory cortex in Proceedings of the National Academy of Sciences, 1998;95:10340-3.

7. Norena AJ, Eggermont JJ (2005). Enriched acoustic environment after noise trauma reduces hearing loss and prevents cortical map reorganization. J Neurosci. 2005;25:699-705.

8. Moore BC, Vinay, Sandhya. The relationship between tinnitus pitch and the edge frequency of the audiogram in individuals with hearing impairment and tonal tinnitus. Hear Res. 2010;26:51-56.

9. Eggermont JJ. Role of auditory cortex in noise and drug-induced tinnitus. Am J Audiol. 2008;27:162-7.

10.Rajan R, Irvine DR (1998). Absence of plasticity of the frequency map in dorsal cochlear nucleus of adult cats after unilateral partial cochlear lesions. J Comp Neurol. 1998;399:35-46.

11.Wang F, Zou L, Hong B, Han D, Range EM, et al. Tonotopic reorganization and spontaneous firing in inferior colliculus during both short and long recovery periods after noise exposure. J Biomed Sci. 20:91.

12. Rouiller EM. Functional organization of the auditory pathways. New York: Oxford University Press; 1997.

13. Markovitz CD, Tang TT, Lim HH. Tonotopic and localized pathways from primary auditory cortex to the central nucleus of the inferior colliculus. Front Neural Circuits. 2013;7:1-11.

14. Suga N. Tuning shifts of the auditory system by corticocortical and corticofugal projections and conditioning. Neurosci Biobehav Rev. 2012;36:969-88.

15.Stebbings KA, Lesicko AMH, Llano DA. The auditory corticocollicular system: Molecular and circuit-level considerations. Hear Res. 2014;314:51-9.

16. Yan J, Suga N. Corticofugal modulation of time-domain processing of biosonar information in bats. Science. 1996;273:1100-3. 
17. Yan J, Suga N. Corticofugal modulation of the midbrain frequency map in the bat auditory system. Nat Neurosci. 1998;1:54-8.

18.Perrot X, Ryvlin P, Isnard J, Guénot M, Catenoix H, Fischer C, et al. Evidence for corticofugal modulation of peripheral auditory activity in humans. Cereb Cortex. 2006;16:907-15.

19. Langers DR, de Kleine E, van Dijk P. Tinnitus does not require macroscopic tonotopic map reorganization. Front Syst Neurosci. 2012;6:114.

20.Bauer CA, Turner JG, Caspary DM, Myers KS, Brozoski TJ. Tinnitus and inferior colliculus activity in chinchillas related to three distinct patterns of cochlear trauma. J Neurosci Res. 2008;86:2564-78.

21. Jastreboff PJ, Sasaki CT. Salicylate-induced changes in spontaneous activity of single units in the inferior colliculus of the guinea pig. Journal of the Acoustical Society of America, 1986;80:1384-91.

22. Wang H, Brozoski TJ, Turner JG, Ling L, Parrish JL, Hughes LF, et al. Plasticity at glycinergic synapses in dorsal cochlear nucleus of rats with behavioral evidence of tinnitus. Neuroscience. 2009;164:747-59.

23. Schaette R, McAlpine D. Tinnitus with a normal audiogram: physiological evidence for hidden hearing loss and computational model. J Neurosci. 2011;31:13452-7.

24.Warr WB. Parallel ascending pathways from the cochlear nucleus: Neural anatomical evidence of functional specialization. Contributions to Sensory Physiology. 1982;7:1-38.

25. Turrigiano GG. Homeostatic plasticity in neuronal networks: the more things change, the more they stay the same. Trends Neurosci. 1990;22:221-7.

26.Xie R, Manis PB. Glycinergic synaptic transmission in the cochlear nucleus of mice with normal hearing and age-related hearing loss. $J$ Neurophysiol. 2013;110:1848-59.

27. Brozoski TJ, Bauer CA, Caspary DM. Elevated fusiform cell activity in the dorsal cochlear nucleus of chinchillas with psychophysical evidence of tinnitus. J Neurosci. 2002;22:2383-90.

28. Barnea G, Attias J, Gold S, Shahar A. Tinnitus with normal hearing sensitivity: extended high-frequency audiometry and auditory-nerve brainstem-evoked responses. Audiology. 1990;29:36-45.

29. Kujawa SG, Liberman MC. Adding insult to injury: cochlear nerve degeneration after "temporary" noise-induced hearing loss. J Neurosci. 29:14077-85.

30.Furman AC, Kujawa SG, Liberman MC. Noise-induced cochlear neuropathy is selective for fibers with low spontaneous rates. $J$ Neurophysiol. 2013;110:577-86.

31.Bourien J, Tang Y, Batrel C, Huet A, Lenoir M, Ladrech S, et al. Contributions of auditory nerve fibers to compound action potential of the auditory nerve. J Neurophysiol. 2014;80:1025-39.

32.Liberman MC, Kujawa SG. Hot Topics-Hidden hearing loss: Permanent cochlear-nerve degeneration after temporary noiseinduced threshold shift. The Journal of the Acoustical Society of America, 2014;135:2311.

33.Plack CJ, Barker D, Prendergast G. Perceptual consequences of "hidden" hearing loss. Trends Hear. 2014;18:1-11.

34. Viana LM, O'Malley JT, Burgess BJ, Jones DD, Oliveira CA, Santos F, et al. Cochlear neuropathy in human presbycusis: Confocal analysis of hidden hearing loss in post-mortem tissue. Hear Res. 2015;327:7888.

35.Paul BT, Bruce IC, Roberts LE. Evidence that hidden hearing loss underlies amplitude modulation encoding deficits in individuals with and without tinnitus. Hear Res. 2016;344:170-82.

36. Gilles A, Schlee W, Rabau S, Wouters K, Fransen E, Van de Heyning $P$. Decreased speech-in-noise understanding in young adults with tinnitus. Front Neurosci.2016;10(288):1-14.

37. Maurizi M, Ottaviani F, Paludetti G, Almadori G, Tassoni A. Contributions to the differentiation of peripheral versus central tinnitus via auditory brainstem response evaluation. Audiology. 1985;24:207-16.

38. Lowe AS, Walton JP. Alteraions in peripheral and central components of the auditory brainstem response: a neural assay of tinnitus. PLoS One. 2015;10(2):e0117228

39. Moore EJ. Bases of auditory brainstem evoked responses. New York: Grune \& Stratton; 1983.

40. Okamoto H, Stracke H, Stoll W, Pantev C. Listening to tailor-made notched music reduces tinnitus loudness and tinnitus related auditory cortex activity. Proceedings of National Academy of Sciences, 2010;107:1207-10.

41.Carhart R, Jerger. Preferred method for clinical determination of pure tone thresholds. Journal of Speech and Hearing Disorders. 1959;24:330-45.

42. Newman CW, Jacobson GP, Spitzer JB. (1996). Development of the Tinnitus Handicap Inventory. Arch Otolaryngol Head Neck Surg. 1996;122:143-8.

43. Jasper HH. Report of the committee on methods of clinical examination in electroencephalography: 1957. Electroencephalography and Clinical Neurophysiology. 1958;10:370-5.

44. Gafni M, Sohmer H, Weizman Z, Robinson MJ. Analysis of auditory nerve brainstem response (ABR) in neonates and very young infants. Arch Otorhinolaryngol.1980;229:167-74.

45. Luczak A, Barth'o P, Harris KD. Spontaneous events outline the realm of possible sensory responses in neocortical populations. Neuron, 2009;62:413-25.

46. Turner JG, Brozoski TJ, Bauer CA, Parrish JL, Myers K, Hughes LF, et al. Gap detection deficits in rats with tinnitus: a potential novel screening tool. Behav Neurosci. 2006;120:188-95.

47. Landis C, Hunt WA, Strauss H. The startle pattern. New York: Farrar \& Rinehart; 1939.

48. Hoffman HS, Searle JL. Acoustic variables in the modification of startle reaction in the rat. J Comp Physiol Psychol. 1965;60:53-8.

49.Ison JR. Temporal acuity in auditory function in the rat: Reflex inhibition by brief gaps in noise. Journal of Comparative Physiological psychology, 1982;96:945-54.

50. Shadwick K, Sun W. Acoustic startle reflex and pre-pulse inhibition in tinnitus patients. Journal of Otology. 2014;9:141-5.

51.Boyen K, Baskent D, van Dijk P. The gap detection test: can it be used to diagnose tinnitus? Ear Hear. 2015;36:e138-45.

52. Campolo J, Lobarinas E, Salvi R. Does tinnitus "fill in" the silent gaps? Noise Health. 2013;15:398-405.

53. Fournier P, Hebert S. Gap detection deficits in humans with tinnitus as assessed with the acoustic startle paradigm: Does tinnitus fill in the gap? Hear Res. 2013;295:16-23.

54.Davis M, Parisi T, Gendelman DS. Habituation and sensitization of startle reflexes elicited electrically from the brainstem. Science. 1982;218:688-90.

$55 . Z$ schorlich VR, Kohling R. How thoughts give rise to actionConscious motor intention increases the excitability of target-specific motor circuits. PLoS One. 2013;8:e83845.

56. Hickox AE, Liberman MC. Is noise-induced cochlear neuropathy key to the generation of hyperacusis of tinnitus? J Neurophysiol. 2014;111:552-64.

57.Gu JW, Herrmann BS, Levine RA, Melcher JR. Brainstem auditory evoked potentials suggest a role for the ventral cochlear nucleus in tinnitus. J Assoc Res Otolaryngol. 2012;13:819-33.

58. Guest $\mathrm{H}$, Munro KJ, Plack CJ. Tinnitus with a normal audiogram: Relation to noise exposure but no evidence for cochlear synaptopathy. Hear Res. 2017;344:265-74.

59.Earl BR, Chertoff ME. Mapping auditory nerve firing density using high-level compound action potentials and high-pass noise masking. J Acoust Soc Am. 2012;131:337-52.

60.Zhao HB, Mei L, Zhu Y. A simple method to detect noise-induced hidden hearing loss. Poster session presented at the Society for Neuroscience 2016 Annual Meeting, San Diego, CA; 2016. 